\title{
GAS CHROMATOGRAPHY-MASS SPECTROSCOPY ANALYSIS OF ROOT OF AN ECONOMICALLY IMPORTANT PLANT, CENCHRUS CILIARIS L. FROM THAR DESERT, RAJASTHAN (INDIA)
}

\author{
SUNITA ARORA*, GANESH KUMAR, SONAM MEENA
}

Department of Botany, Jai Narain Vyas University, Jodhpur, Rajasthan, India. Email: jnvusunitarora@gmail.com

Received: 18 April 2017, Revised and Accepted: 27 May 2017

ABSTRACT

Objective: The study was carried out with an objective to characterize the possible bioactive phytochemical constituents from the root of Cenchrus ciliaris L. using various solvents of different polarities, i.e., methanol, ethyl acetate, and hexane by gas chromatography-mass spectroscopy (GC-MS) analysis.

Methods: Plant material was collected from harsh, xeric conditions of "Thar" during August to October. The shade-dried plant root powder was extracted with solvents using Soxhlet extractor. The phytochemical compounds were investigated using PerkinElmer GC-MS, while the mass spectra of the compounds found in the extract was matched with the National Institute of Standards and Technology and Willey 8 library.

Results: Maximum \% area is found for stigmasta-5,22-dien-3-ol and present in maximum amount (12.68\%) with reaction time (RT)=36.461 minutes in the methanolic extract. 1,2,3-propanetriol, 1-acetate is present in maximum amount (13.15\%) with RT=6.582 minutes in the ethyl acetate extract. Tetracontane is present in maximum amount (16.70\%) with RT=18.744 minutes in the hexane extract of root of $C$. ciliaris $\mathrm{L}$.

Conclusions: Green plants synthesize and preserve a variety of biochemical compounds. Plant secondary metabolites are commercially important and are used by pharmaceutical industry as well as the traditional practitioners. The GC-MS study helps to predict the formula and structure of phytoconstituents that can be used for drug design, and further investigation may lead to the development of various drug formulations.

Keywords: Cenchrus ciliaris L., Gas chromatography-mass spectroscopy, Secondary metabolites, Pharmaceutical, Polarity.

(C) 2017 The Authors. Published by Innovare Academic Sciences Pvt Ltd. This is an open access article under the CC BY license (http://creativecommons. org/licenses/by/4. 0/) DOI: http://dx.doi.org/10.22159/ajpcr.2017.v10i8.19259

\section{INTRODUCTION}

Cenchrus ciliaris L. (Poaceae) is one of the important forage plants, commonly known as "Dhaman grass." It is perennial $C_{4}$ grass with a deep, adventitious root system and tough culms that are erect to somewhat prostrate branched with swollen bases. Leaves are basal, cauline with sharp blades arranged at nodes, rough textured, and blades have prominent midribs with keeled leaf sheaths. The inflorescence is erect, dense, cylindrical, and spike-like. The rachis bears lanceolate spikelets enclosed by diamond-shaped involucres of prickly bristles. Seeds are obovoid and of caryopsis type.

The plant is the most suitable and highly nutritive grass for environmental conditions of desert gaining attention in the various fields of research. It is more competitive under the conditions of high temperature, solar radiation, and low moisture [1]. These grasses are more efficient at gathering $\mathrm{CO}_{2}$ and utilizing nitrogen from the atmosphere and for recycling $\mathrm{N}$ in the soil. Grasses have an excellent soil binding capacity which helps to conserve soil in the desert areas [2]. Dry hot summers and pleasant dry winters are prominent features of the Thar desert. The mean daily maximum temperature in summer ranges from $41^{\circ} \mathrm{C}$ to $46^{\circ} \mathrm{C}$ and can reach up to $50^{\circ} \mathrm{C}$ during the hot summer noon. Rainfall is sparse, ranging from 127 to $254 \mathrm{~mm}$ annually, and is confined mainly to the rainy season [3].

Earlier reports suggest that $C$. ciliaris $\mathrm{L}$. is a potential grass of "Thar desert." However, to date, there are no reports regarding exploitation of root with methanol, ethyl acetate, and hexane as solvents.

\section{METHODS}

Collection and processing of plant material

Fresh plants free from diseases were collected from Jodhpur district, Rajasthan, India, during rainy season from August to October. Plants were identified and authenticated by Botanical Survey of India, Jodhpur. Fresh roots were incised, washed, air dried, and powdered in an electric grinder. The coarse powdered plant material was stored in airtight containers.

Soxhlet assembly was used to prepare root extract [4] in various solvents (methanol, ethyl acetate, and hexane). Repeated extraction was done until a colorless extract was obtained. All the plant extracts under study were evaporated to dryness and stored at $4^{\circ} \mathrm{C}$ for future use. The gas chromatography-mass spectroscopy (GC-MS) analysis was performed at Advanced Instrumentation Research Facility (AIRF), Jawaharlal Nehru University, New Delhi, India.

\section{Identification of phytocomponents}

Identification of phytocomponents was done using Willey 8 and National Institute of Standards and Technology library that remains attached to the GC-MS. Retention indices of analyzed compounds were compared with stored components, and the phytoconstituents were identified as per their structure, $\%$ area, molecular formula, and spectrum. Phytoconstituents exhibit a broad spectrum of effects. Some may be beneficial and used for the treatment of various diseases while others may be toxic [5].

\section{RESULTS AND DISCUSSION}

GC-MS analysis of the root extracts of $C$. ciliaris in solvents with different polarities, i.e., methanol, ethyl acetate, and hexane revealed 46, 70, and 80 peaks in chromatogram (Figs. 1-3) indicating the presence of 42,64 , and 71 active compounds, respectively. Confirmation of these compounds was based on their retention time, molecular formula, and molecular weight. These bioactive compounds show various biological activities that have been reported earlier. Medicinal plant-based drugs exhibit several broad spectrum activities [6] 
Table 1: Bioactivity of compounds identified in methanol root extract

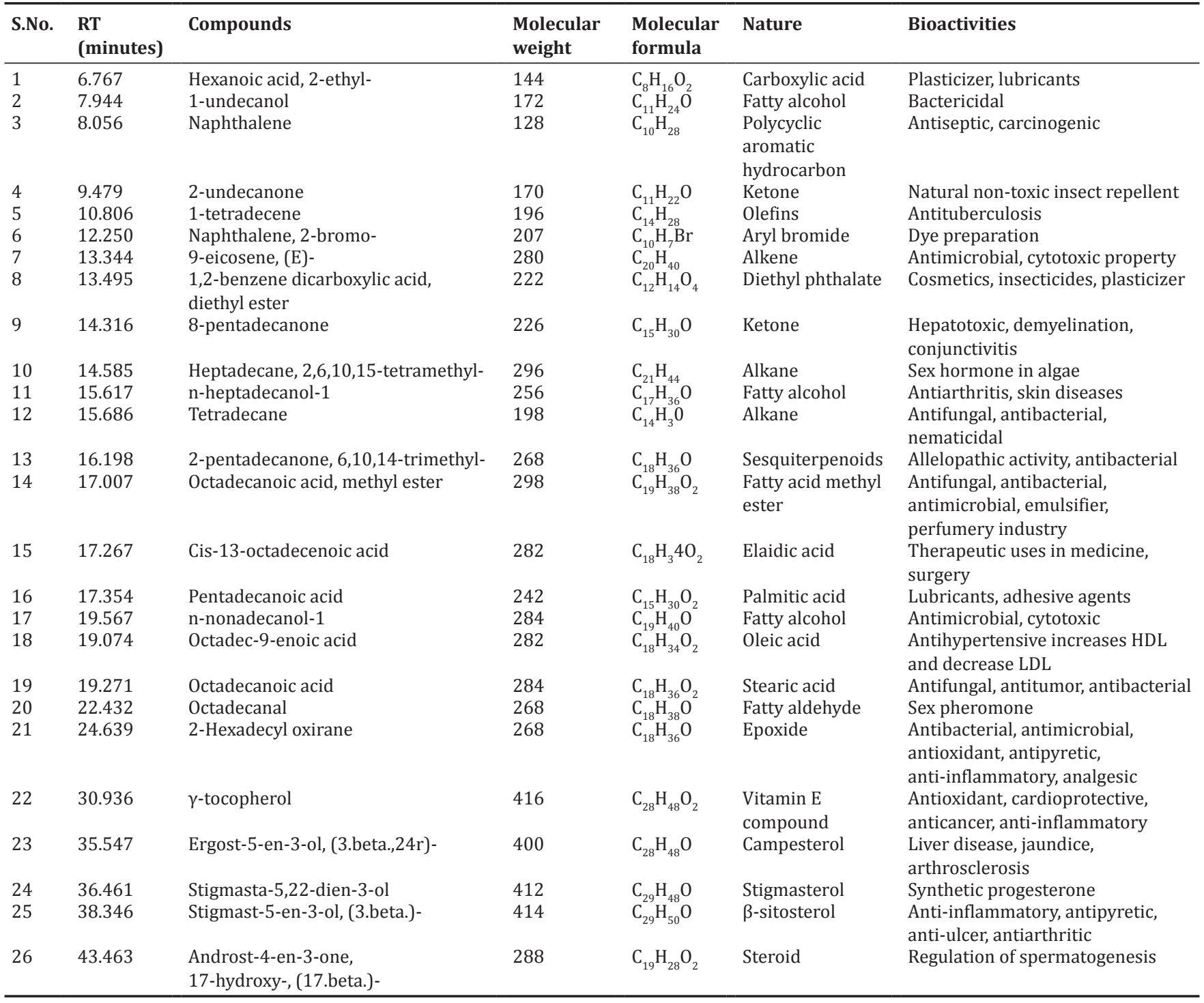

RT: Reaction time

Table 2: Bioactivity of compounds identified in ethyl acetate root extract

\begin{tabular}{|c|c|c|c|c|c|c|}
\hline S.No. & $\begin{array}{l}\text { RT } \\
\text { (minutes) }\end{array}$ & Compounds & $\begin{array}{l}\text { Molecular } \\
\text { weight }\end{array}$ & $\begin{array}{l}\text { Molecular } \\
\text { formula }\end{array}$ & Nature & Bioactivity \\
\hline 1 & 6.582 & 1,2,3-propanetriol, 1 -acetate & 134 & $\mathrm{C}_{5} \mathrm{H}_{10} \mathrm{O}_{4}$ & Monoacetin & Antiadipogenic \\
\hline 2 & 8.056 & Naphthalene & 128 & $\mathrm{C}_{10}^{5} \mathrm{H}_{8}$ & $\begin{array}{l}\text { Polycyclic } \\
\text { aromatic } \\
\text { hydrocarbon }\end{array}$ & Antiseptic, carcinogenic \\
\hline 3 & 10.909 & Tetradecane & 198 & $\mathrm{C}_{14} \mathrm{H}_{30}$ & Alkane & Antismicrobial, \\
\hline 4 & 12.206 & Pentadecane & 212 & $\mathrm{C}_{15}^{14} \mathrm{H}_{32}^{30}$ & Alkane & $\begin{array}{l}\text { Suger-phosphatase inhibitor, } \\
\text { chymosin inhibitor, antibacterial }\end{array}$ \\
\hline 5 & 12.546 & 1-tridecanol & 200 & $\mathrm{C}_{13} \mathrm{H}_{28} \mathrm{O}$ & Fatty alcohol & Natural mosquito control agent \\
\hline 6 & 13.430 & Hexadecane & 226 & $\mathrm{C}_{16} \mathrm{H}_{34}^{20}$ & Alkane & Antifungal, antibacterial, antioxidant \\
\hline 7 & 13.498 & $\begin{array}{l}\text { 1,2-benzene dicarboxylic acid, diethyl } \\
\text { ester }\end{array}$ & 222 & $\mathrm{C}_{12} \mathrm{H}_{14}^{34} \mathrm{O}_{4}$ & $\begin{array}{l}\text { Diethyl } \\
\text { phthalate }\end{array}$ & Cosmetics, insecticides, plasticizer \\
\hline 8 & 14.173 & 2-methyl tetracosane & 352 & $\mathrm{C}_{25} \mathrm{H}_{52}$ & Alkane & Free radical scavenger \\
\hline 9 & 14.264 & Heptadecane, 2,6,10,15-tetramethyl- & 296 & $\mathrm{C}_{21}^{25} \mathrm{H}_{44}^{52}$ & Alkane & Sex hormone in algae \\
\hline 10 & 14.589 & Heptadecane & 240 & $\mathrm{C}_{17}^{21} \mathrm{H}_{36}^{44}$ & Alkane & Antioxidant \\
\hline 11 & 14.657 & Pentadecane, 2,6,10,14-tetramethyl- & 268 & $\mathrm{C}_{19}^{1} \mathrm{H}_{40}$ & Alkane & $\begin{array}{l}\text { Pathogenesis of rheumatoid arthritis } \\
\text { and lupus }\end{array}$ \\
\hline 12 & 15.224 & Eicosane & 282 & $\mathrm{C}_{20} \mathrm{H}_{42}$ & Alkane & $\begin{array}{l}\text { Antifungal, antitumor, antibacterial, } \\
\text { larvicidal, antimicrobial, cytotoxic }\end{array}$ \\
\hline 13 & 15.291 & Tricosane & 324 & $\mathrm{C}_{23} \mathrm{H}_{48}$ & Alkane & Antibacterial \\
\hline 14 & 15.383 & Heptadecane, 3-methyl- & 254 & $\mathrm{C}_{18}^{25} \mathrm{H}_{38}^{40}$ & Alkane & Pest repellent, sex pheromone \\
\hline
\end{tabular}


(Table 2: Continued)

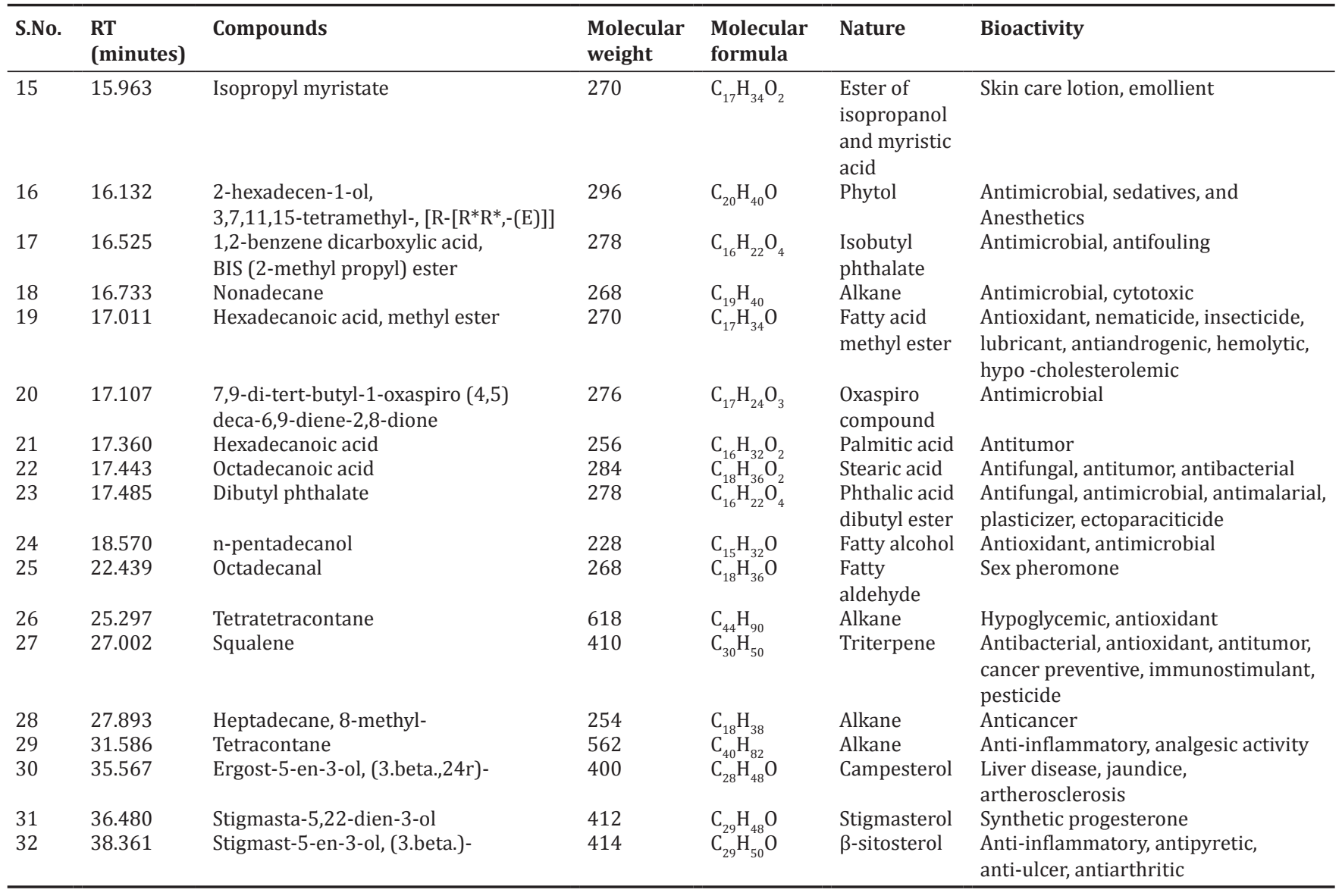

RT: Reaction time

Table 3: Bioactivity of compounds identified in hexane root extract

\begin{tabular}{|c|c|c|c|c|c|c|}
\hline S.No. & $\begin{array}{l}\text { RT } \\
\text { (minutes) }\end{array}$ & Compound & $\begin{array}{l}\text { Molecular } \\
\text { weight }\end{array}$ & $\begin{array}{l}\text { Molecular } \\
\text { formula }\end{array}$ & Nature & Bioactivity \\
\hline 1 & 9.527 & Tridecane & 184 & $\mathrm{C}_{13} \mathrm{H}_{28}$ & Alkane & Fragrance agent \\
\hline 2 & 9.643 & 2-isopropyl-5-methyl-1-heptanol & 172 & $\mathrm{C}_{11}^{13} \mathrm{H}_{24}^{28} \mathrm{O}$ & $\begin{array}{l}\text { Alcoholic } \\
\text { compound }\end{array}$ & Antimicrobial \\
\hline 3 & 9.908 & Octadecane & 254 & $\mathrm{C}_{18} \mathrm{H}_{38}$ & Alkane & Lubricants, anticorrosion agents \\
\hline 4 & 10.906 & Tetradecane & 198 & $\mathrm{C}_{14}^{18} \mathrm{H}_{30}^{38}$ & Alkane & Antifungal, antibacterial \\
\hline 5 & 12.204 & Pentadecane & 212 & $\mathrm{C}_{15}^{14} \mathrm{H}_{32}^{30}$ & Alkane & $\begin{array}{l}\text { Suger-phosphatase inhibitor, } \\
\text { chymosin inhibitor, antibacterial }\end{array}$ \\
\hline 6 & 12.736 & Eicosane & 282 & $\mathrm{C}_{20} \mathrm{H}_{42}$ & Alkane & $\begin{array}{l}\text { Antifungal, antitumor, } \\
\text { antibacterial, larvicidal, cytotoxic, } \\
\text { antimicrobial }\end{array}$ \\
\hline 7 & 13.428 & Hexadecane & 226 & $\mathrm{C}_{16} \mathrm{H}_{34}$ & Alkane & $\begin{array}{l}\text { Antifungal, antibacterial, } \\
\text { antioxidant }\end{array}$ \\
\hline 8 & 14.107 & Pyrrolidine & 71 & $\mathrm{C}_{4} \mathrm{H}_{9} \mathrm{~N}$ & $\begin{array}{l}\text { Cyclic secondary } \\
\text { amine }\end{array}$ & $\begin{array}{l}\text { Antimicrobial, antitumor, } \\
\text { anti-HIV-1, anticonvulsant }\end{array}$ \\
\hline 9 & 14.170 & 2-methyl tetracosane & 352 & $\mathrm{C}_{25} \mathrm{H}_{52}$ & Alkane & Free radical scavenger \\
\hline 11 & 14.587 & Heptadecane & 240 & $\mathrm{C}_{17} \mathrm{H}_{36}$ & Alkane & Antioxidant \\
\hline 12 & 14.656 & Pentadecane, 2,6,10,14-tetramethyl- & 268 & $\mathrm{C}_{19}^{17} \mathrm{H}_{40}^{36}$ & Pristane & $\begin{array}{l}\text { Pathogenesis of rheumatoid } \\
\text { arthritis and lupus }\end{array}$ \\
\hline 13 & 14.737 & Tricosane & 324 & $\mathrm{C}_{23} \mathrm{H}_{48}$ & Alkane & Antibacterial \\
\hline 14 & 14.795 & Pentadecanal & 226 & $\mathrm{C}_{15}^{23} \mathrm{H}_{30} \mathrm{O}$ & Fatty aldehyde & $\begin{array}{l}\text { Nutrient stabilizers, surfactants, } \\
\text { and emulsifier }\end{array}$ \\
\hline 15 & 15.073 & 1-dodecanol, 2-octyl- & 298 & $\mathrm{C}_{20} \mathrm{H}_{42} \mathrm{O}$ & Branched alcohol & $\begin{array}{l}\text { Emollients, perfuming agents, } \\
\text { cosmetics }\end{array}$ \\
\hline 16 & 15.217 & Hexadecane, 2,6,10,14-tetramethyl- & 282 & $\mathrm{C}_{20} \mathrm{H}_{42}$ & Phytane & Biomarkers in petroleum studies \\
\hline
\end{tabular}


(Table 3: Continued)

\begin{tabular}{|c|c|c|c|c|c|c|}
\hline S.No. & $\begin{array}{l}\text { RT } \\
\text { (minutes) }\end{array}$ & Compound & $\begin{array}{l}\text { Molecular } \\
\text { weight }\end{array}$ & $\begin{array}{l}\text { Molecular } \\
\text { formula }\end{array}$ & Nature & Bioactivity \\
\hline 17 & 15.265 & Tetradecanoic acid & 228 & $\mathrm{C}_{14} \mathrm{H}_{28} \mathrm{O}_{2}$ & Myristic acid & $\begin{array}{l}\text { Antioxidant, anticancer, } \\
\text { hypocholesterolemic }\end{array}$ \\
\hline 18 & 15.685 & Nonadecane & 268 & $\mathrm{C}_{19} \mathrm{H}_{40}$ & Alkane & Antimicrobial, cytotoxic \\
\hline 19 & 15.958 & Isopropyl myristate & 270 & $\mathrm{C}_{17}^{19} \mathrm{H}_{34}^{40} \mathrm{O}_{2}$ & $\begin{array}{l}\text { Ester of fatty acid } \\
\text { alcohol }\end{array}$ & Skin care lotion, emollient \\
\hline 20 & 16.200 & 2-penta decanone, 6,10,14-trimethyl- & 268 & $\mathrm{C}_{18} \mathrm{H}_{36} \mathrm{O}$ & Sesqui-terpenoids & Allelopathic activity \\
\hline 22 & 16.671 & Tetratetracontane & 618 & $\mathrm{C}_{44} \mathrm{H}_{9} \mathrm{O}$ & Alkane & Hypoglycemic, antioxidant \\
\hline 23 & 17.105 & $\begin{array}{l}\text { 7,9-di-tert-butyl-1-oxaspiro }(4,5) \\
\text { deca-6,9-diene-2,8-dione }\end{array}$ & 276 & $\mathrm{C}_{17}^{44} \mathrm{H}_{24} \mathrm{O}_{3}$ & $\begin{array}{l}\text { Oxaspiro } \\
\text { compound }\end{array}$ & Antimicrobial \\
\hline 24 & 17.406 & Pentadecanoic acid & 242 & $\mathrm{C}_{15} \mathrm{H}_{30} \mathrm{O}_{2}$ & $\begin{array}{l}\text { Saturated fatty } \\
\text { acid }\end{array}$ & Lubricant, Adhesive agents \\
\hline 25 & 18.333 & Heptadecanoic acid & 270 & $\mathrm{C}_{17} \mathrm{H}_{34} \mathrm{O}_{2}$ & Margaric acid & Antimicrobial \\
\hline 26 & 18.744 & Tetracontane & 562 & $\mathrm{C}_{40} \mathrm{H}_{82}^{34}$ & Alkane & Anti-inflammatory \\
\hline 28 & 19.470 & $\begin{array}{l}2,6,10,15,19,23 \text {-hexamethyl } \\
\text { tetracosane }\end{array}$ & 332 & $\mathrm{C}_{30} \mathrm{H}_{62}$ & Squalane & $\begin{array}{l}\text { Antibacterial, antioxidant, } \\
\text { antitumor, anticancer, } \\
\text { immunostimulant }\end{array}$ \\
\hline 29 & 20.616 & n-nonadecanol-1 & 284 & $\mathrm{C}_{19} \mathrm{H}_{40} \mathrm{O}$ & Fatty alcohol & Antimicrobial, cytotoxic \\
\hline 30 & 21.517 & Eicosanoic acid & 312 & $\mathrm{C}_{20} \mathrm{H}_{40} \mathrm{O}_{2}$ & Arachidic acid & Anticancer, anti-inflammatory \\
\hline 31 & 23.202 & 1-heptacosanol & 396 & $\mathrm{C}_{27} \mathrm{H}_{56} \mathrm{O}^{2}$ & Fatty alcohol & $\begin{array}{l}\text { Nematicidal, anticancer, } \\
\text { antioxidant, antimicrobial }\end{array}$ \\
\hline 32 & 23.490 & $\begin{array}{l}\text { Hexadecanoic acid, } \\
\text { 2-hydroxy-1-(hydroxyl methyl) ethyl } \\
\text { ester }\end{array}$ & 330 & $\mathrm{C}_{19} \mathrm{H}_{38} \mathrm{O}_{4}$ & Palmitoyl glycerol & Antioxidant \\
\hline 33 & 23.869 & 1,2-benzene dicarboxylic acid & 390 & $\mathrm{C}_{8} \mathrm{H}_{6} \mathrm{O}_{4}$ & Phthalic acid & $\begin{array}{l}\text { Antioxidant, antimicrobial, } \\
\text { antifouling }\end{array}$ \\
\hline 34 & 25.300 & Pentatriacontane & 492 & $\mathrm{C}_{35} \mathrm{H}_{72}$ & Alkane & Antibacterial, antiviral \\
\hline 35 & 25.631 & $\begin{array}{l}\text { Octadecanoic acid, 2,3-dihydroxy } \\
\text { propyl ester }\end{array}$ & 358 & $\mathrm{C}_{21}^{35} \mathrm{H}_{42} \mathrm{O}_{4}$ & 1-glyceryl stearate & Anticancer, antimicrobial \\
\hline 37 & 34.072 & $\begin{array}{l}\text { Cholest-22-ene-21-ol, } \\
\text { 3,5-dehydro-6-methoxy-, pivalate }\end{array}$ & 498 & $\mathrm{C}_{33} \mathrm{H}_{54} \mathrm{O}_{3}$ & Steroid & $\begin{array}{l}\text { Antimicrobial, anti-inflammatory, } \\
\text { antiarthritic, antiuretic, } \\
\text { antiasthmatic }\end{array}$ \\
\hline 38 & 35.588 & Ergost-5-en-3-ol, (3.beta.,24r)- & 400 & $\mathrm{C}_{28} \mathrm{H}_{48} \mathrm{O}$ & Campesterol & $\begin{array}{l}\text { Liver disease, jaundice, } \\
\text { artherosclerosis }\end{array}$ \\
\hline 39 & 36.531 & Stigmasta-5,22-dien-3-ol & 412 & $\mathrm{C}_{29} \mathrm{H}_{48} \mathrm{O}$ & Stigmasterol & Synthetic progesterone \\
\hline 40 & 38.391 & Stigmast-5-en-3-ol, (3.beta.)- & 414 & $\mathrm{C}_{29}^{29} \mathrm{H}_{50}^{48} \mathrm{O}$ & $\beta$-sitosterol & $\begin{array}{l}\text { Anti-inflammatory, antipyretic, } \\
\text { anti-ulcer, antiarthritic }\end{array}$ \\
\hline 41 & 41.285 & Lupeol & 426 & $\mathrm{C}_{30} \mathrm{H}_{50} \mathrm{O}$ & Triterpenoids & $\begin{array}{l}\text { Anti-inflammatory, anticancer, } \\
\text { antiprotozoal, chemopreventive }\end{array}$ \\
\hline 42 & 41.875 & Stigmasta-3,5-dien-7-one & 410 & $\mathrm{C}_{29} \mathrm{H}_{46} \mathrm{O}$ & $\beta$-saccharostenone & $\begin{array}{l}\text { Free radical scavenging, } \\
\text { antidiabetic, anticancer }\end{array}$ \\
\hline 43 & 43.551 & Stigmast-4-en-3-one & 412 & $\mathrm{C}_{29} \mathrm{H}_{48} \mathrm{O}$ & 3-keto-steroid & $\begin{array}{l}\text { Hepatoprotective, antimicrobial, } \\
\text { anti-inflammatory, anticancer }\end{array}$ \\
\hline
\end{tabular}

Phytosterols are cholesterol-like molecules found in plants; the most common phytosterols are stigmasterol, $\beta$-sitosterol, and campesterol [7]. They have been clinically proved to reduce blood cholesterol and scientific reports suggest that they possess anticancerous and antioxidant activity $[8,9]$. Stigmast-5-en-3 $\beta$-ol $(\beta$-sitosterol), a phytosterol shows anti-inflammatory, antipyretic, antiarthritic, antiulcer, insulin-releasing, and estrogenic effects. $\beta$-sitosterol is used for its cholesterol-lowering property [10]. Tetradecane shows antifungal, antibacterial, and nematicidal activity [11]. Octadecanoic acid (stearic acid) shows hypocholesterolemic property and is used in cosmetics, flavor, lubricant, perfumery, and suppository [12]. Stearic acid shows antifungal, antitumor, and antibacterial activity $[13,14]$. The demand for pharmaceuticals and nutraceuticals is growing quickly worldwide, and globally attracting consumers [15].
26 phytochemicals in methanolic extract (Table 1), 32 in ethyl acetate extract (Table 2), and 43 in hexane extract (Table 3) were identified with various biological activities. Stigmasta-5, 22-dien-3-ol, octadecanoic acid, ergost-5-en-ol, (3.beta., 24r)-, stigmast-5-en-3-ol, (3.beta.)-, and tetradecane are common compounds. Mass spectrum of these phytocompounds is shown in Figs. 4-8.

Stigmasta-5, 22-dien-3-ol is present in maximum amount (12.68\%), followed by pentadecanoic acid (11.35\%), stigmast-5-en-3-ol, (3.beta.)(8.50\%), ergost-5-en-3-ol, (3.beta.,24r)- (7.08\%), and octadec-9-enoic acid $(4.55 \%)$ in methanolic extract. 1,2,3-propanetriol, 1-acetate is present in maximum amount (13.15\%), followed by stigmasta5,22-dien-3-ol (6.45\%), hexadecanoic acid (5.54\%), ergost-5-en-3ol, (3.beta.,24r)- (4.43\%), and tetracontane (3.73\%) in ethyl acetate 


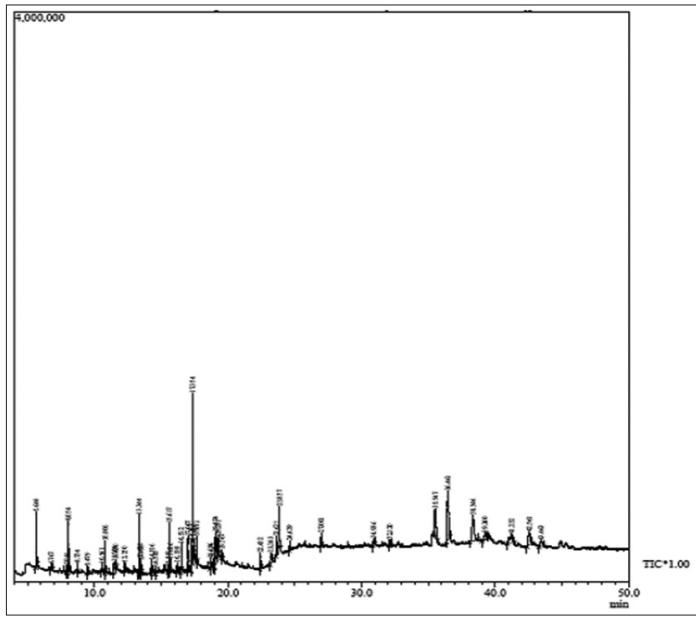

Fig. 1: Gas chromatography-mass spectroscopy chromatogram of the methanol extract of root of Cenchrus ciliaris $\mathrm{L}$.

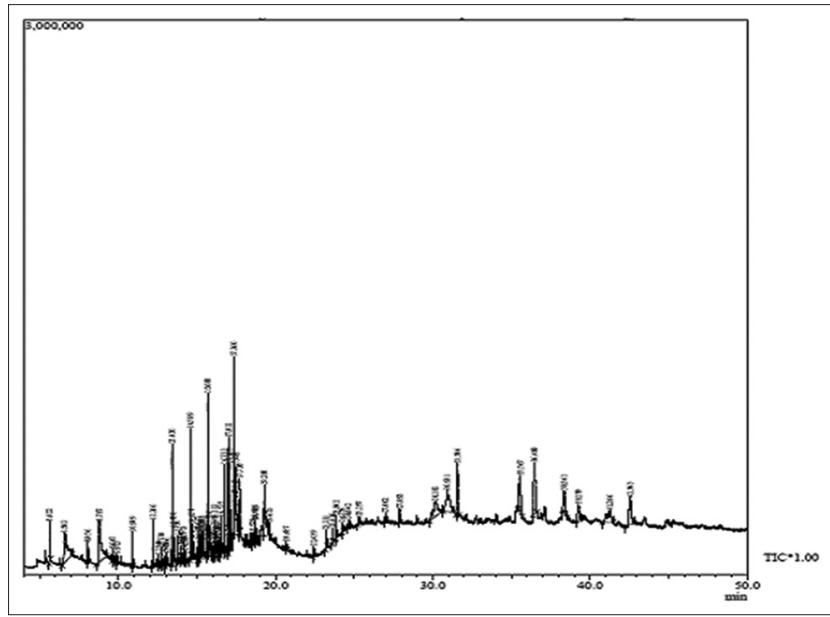

Fig.2: Gas chromatography-mass spectroscopy chromatogram of the ethyl acetate extract of root of Cenchrus ciliaris L.

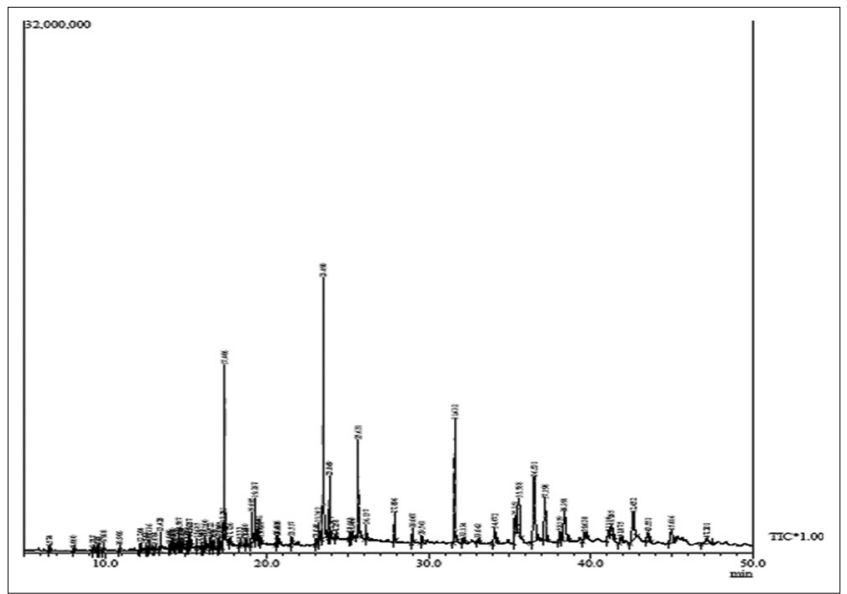

Fig. 3: Gas chromatography-mass spectroscopy chromatogram of the hexane extract of root of Cenchrus ciliaris $\mathrm{L}$.

extract. Tetracontane is present in maximum amount (16.70\%), followed by hexadecanoic acid, 2-hydroxy-1-(hydroxymethyl) ethyl ester (14.96\%), stigmasta-5,22-dien-3-ol (9.34\%), pentadecanoic acid (7.84\%), and octadecanoic acid, 2,3-dihydroxypropyl ester (6.56\%) in hexane extract of $C$. ciliaris.

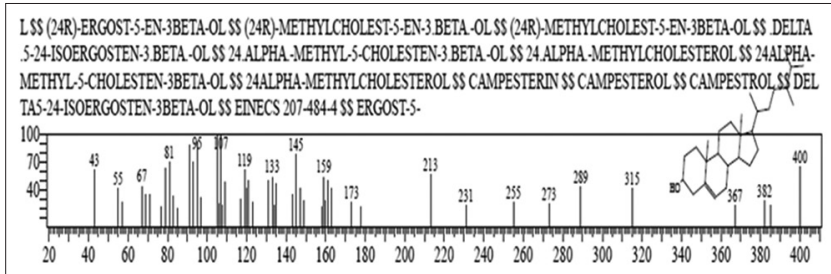

Fig. 4: Mass spectrum of ergost-5-en-3-ol, (3.beta., 24r)-

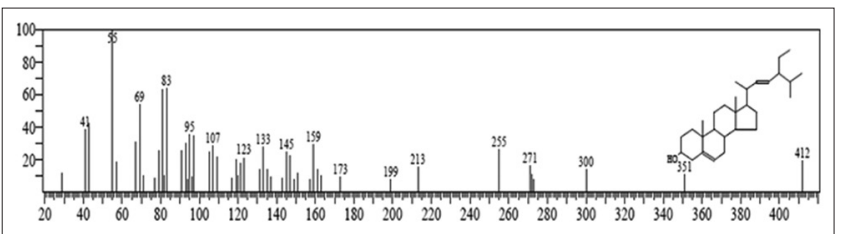

Fig. 5: Mass spectrum of stigmasta-5,22-dien-3-ol

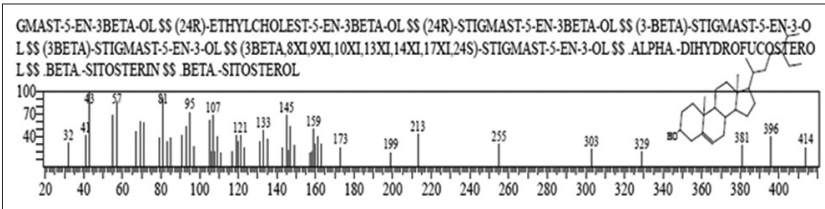

Fig. 6: Mass spectrum of stigmast-5-en-3-ol, (3.beta.)-

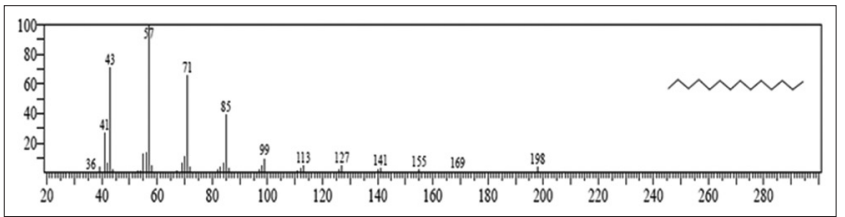

Fig. 7: Mass spectrum of tetradecane

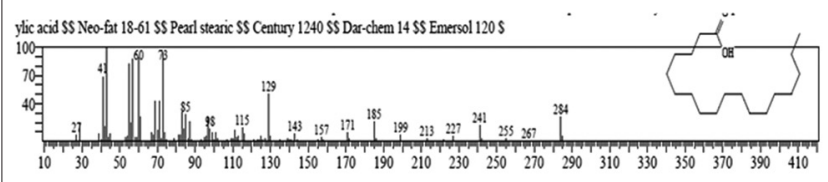

Fig. 8: Mass spectrum of octadecanoic acid

\section{CONCLUSION}

GC-MS study has exploited the potential of C. ciliaris. This plant can be a good source of phytoconstituents such as alkane, carboxylic acid, phytosterol, aldehyde, ketone, fatty acid ester, fatty alcohol, and terpenes. Ergost-5-en-3-ol, (3.beta., 24r)- (campesterol) and stigmasta-5, 22-dien-3-ol (stigmasterol).

We are first to analyze these compounds from this forage plant using hexane like solvents of high polarity. This study suggests that solvents varying in polarity can extract various amounts of phytochemicals. Root extracts in hexane eluted highest number of compounds as compared to methanol and ethyl acetate extracts. The analysis suggests that solvent-linked polarity and extraction potentiality are the best tool for extraction of bioactive compounds. Ethico-legal issues require further discussions and considerations before practical use.

\section{ACKNOWLEDGMENT}

The authors would like to grateful to the Department of Botany, JNV University, Jodhpur and AIRF, Jawaharlal Nehru University, New Delhi, for providing infrastructure and instrumentation facilities. 


\section{REFERENCES}

1. Agrawal P. Ecophysiological and Biochemical Studies Related to Drought Adaptation in Grasses of Indian Desert. Ph.D. Thesis. Jodhpur: JN Vyas University; 2007.

2. Sinha RK, Bhatia S, Vishnoi R. Desertification Control and Rangeland Management in the Thar Desert of India. RALA Report No. 200; 1996. p. $115-23$

3. Arora J, Goyal S, Ramawat KG, editors. Biodiversity, biology and conservation of medicinal plants of the Thar Desert. Desert Plants, Biology and Biotechnology. Berlin, Heidelberg: Springer-Verlag; 2010. p. 3-36.

4. Harborne JB, editors. Methods of plant analysis. Phytochemical Methods. $2^{\text {nd }}$ ed. London: Chapman and Hall; 1984. p. 5-6.

5. Katiyar D, Singh V, Ali M. Isolation, characterization and prediction of the biological activity of two new fatty esters and a phenol from the heartwood of Pterocarpus marsupium Roxb. Int J Pharm Pharm Sci 2017;9(2):117-22.

6. Sermakkani M, Thangapandian V. GC-MS analysis of Cassia itallica leaf methanol extract. Asian J Pharm Clin Res 2012;5(2):90-4.

7. Baker DH, Ibrahim EA, Kandeil A, Baz FK. Sterols bioactivity of Ruta graveolens L. And Murraya paniculata L. Int J Pharm Pharm Sci 2017;9(2):103-8
8. Bradford PG, Awad AB. Phytosterols as anticancer compounds. Mol Nutr Food Res 2007;51(2):161-70.

9. Zawistowski J. Tangible health benefits of phytosterol functional foods. In: Smith J, Charter E, editors. Functional Food Product Development. Oxford, UK: Wiley Blackwell; 2010. p. 362-72.

10. Patra A, Jha S, Murthy PN, Manik A, Sharone A. Isolation and characterization of stigmast - 5-en-3 $\beta$-ol ( $\beta$-sitosterol) from the leaves of Hygrophila spinosa T. Anders Int J Pharm Sci Res 2010;1(2):95-100

11. Seo SM, Kim J, Kim E, Park HM, Kim YJ, Park IK, et al. Structureactivity relationship of aliphatic compounds for nematicidal activity against pine wood nematode (Bursaphelenchus xylophilus). J Agric Food Chem 2010;58(3):1823-7.

12. Markkas N, Govindharajalu M. Determination of phytocomponents in the methanolic extract of Mollugo cerviana by GC-MS analysis. Int J Res Biol Sci 2015;5(4):26-9.

13. Vijisaral ED, Subramanian A. GC - MS analysis of ethanol extract of Cyperus rotundus leaves. Int J Curr Biotechnol 2014;2(1):19-23.

14. Akpuaka A, Ekwenchi MM, Dashak DA, Dildar A. Biological activities of characterized isolates of n-hexane extract of Azadirachta indica A. Juss (Neem) Leaves. Nat Sci 2013;11(5):141-7.

15. Bhattacharjee M. Pharmaceutically valuable bioactive compounds of algae. Asian J Pharm Clin Res 2016;9(6):43-7. 